\title{
Optoelectronic and thermal properties of boron-bismuth compound
}

\author{
Salah Daoud $^{1 *}$, Nadhira Bioud ${ }^{2}$, Noudjoud Labgaa ${ }^{2}$, Rabie Mezouar ${ }^{1}$ \\ ${ }^{1}$ Faculté des Sciences et de la Technologie, Université de Bordj Bou Arreridj, 34000, Algeria \\ ${ }^{2}$ Laboratoire d'Optoélectronique et Composants, Université Ferhat Abbes-Sétif, 19000, Algeria \\ *Corresponding author_E-mail: salah_daoud07@yahoo.fr
}

Copyright $\odot 2014$ Salah Daoud et al. This is an open access article distributed under the Creative Commons Attribution License, which permits unrestricted use, distribution, and reproduction in any medium, provided the original work is properly cited.

\begin{abstract}
In the present work, we report first principles calculations of the near-neighbor distance (bond length) and the average energy gap using the pseudopotential plane wave method, in the framework of the density functional theory (DFT) within the local density approximation (LDA) and the Hartwigzen-Goedecker-Hutter (HGH) scheme for the pseudopotential of Boron- Bismuth compound in its structure zincblende phase.

The refractive index, the plasmon energy, the force constants, the lattice energy, the homopolar and heteropolar energies, the ionicity, the linear optical susceptibility, the hardness, the dielectric constants, the Debye temperature and the melting temperature are then predicted by mean of some simple emperical formulas. The results obtained are analyzed and compared with the available theoretical data of the literature
\end{abstract}

\section{Introduction}

Many $\mathrm{A}^{\mathrm{III}} \mathrm{B}^{\mathrm{V}}$ semiconductor compounds crystallize usually in the cubic zincblende (B3) structure. In the cubic zincblende structure, the atoms are tetrahedrally bonded to four nearest-neighbors usually through covalent bonds [1]. Some important remarkable differences between the physical properties of conventional III-V and the heavier bismuth III-V compounds were noticed recently in the work of Zaoui et al [2].

The electronic behavior of Boron-bismuth (BBi) in its zincblende phase was studied by Madouri et al [3], using the full potential linearized augmented plane wave (FPLAPW) method and as implemented in the WIEN2k code [4]. Under hydrostatic pressure, the low pressure phase of crystalline material is destabilized and structural phase transition occurs. Unfortunately, to the best of our knowledge, there are very little results [5-7] on the phase transition pressure for (B3) $\mathrm{BBi}$ compound. The phase transition from the zincblende (B3) to Rock-salt (B1) phase appears between 24.5GPa [5] and $34 \mathrm{GPa}[7]$.

In the present work, we report first principles calculations of the bond length and the average energy gap using the pseudopotential plane wave method, in the framework of the density functional theory (DFT) within the local density approximation (LDA).

The refractive index, the plasmon energy, the force constants, the lattice energy, the homopolar and heteropolar energies, the ionicity, the linear optical susceptibility, the hardness, the dielectric constants, the Debye temperature and finally the melting temperature of (B3) BBi compound are obtained by using only a simple emperical formulas. Our results of different parameters obtained are analyzed and compared with the available data of the literature.

\section{Computational methods}

First-principles calculations were implemented through the ABINIT code [9]. ABINIT code computer is a common project of the Université Catholique de Louvain, Corning Incorporated and other contributors. The exchange-correlation functions were treated within the local density approximation (LDA) using the Teter and Pade parameterization [10]. 
The Hartwigzen-Goedecker-Hutter (HGH) scheme [11] was employed for the pseudopotential. A plane-wave basis set was used to solve the Kohn-Sham equations in the pseudopotential implementation of the DFT-LDA.

The Brillouin zone integrations were replaced by discrete summations over a special set of k-points using the standard k-point technique of Monkhorst and Pack [12], where the k-point mesh used is $(6 \times 6 \times 6)$. The plane-wave energy cutoff to expand the wave functions is set to 90 Hartree. Careful convergence tests show that with these parameters relative energy converged to better than $10^{-5}$ Hartree.

\section{Results and discussions}

\subsection{Refractive index, plasmon energy, force constants and lattice energy}

The simple definition of the refractive index of a transparent optical medium is the ratio of the velocity of light in vacuum on the phase velocity $v_{\mathrm{ph}}$ of the transparent optical medium. It can be also calculated from the relative permittivity $\varepsilon$ and the relative permeability $\mu$ of the optical medium $n^{2}=\varepsilon$. $\mu$. The evaluation of refractive indices of a semiconductor is of considerable importance for the fabrication of devices in optoelectronic applications.

Reddy et al. [13] and Kumar and Singh [14] frequent attempts have been made to understand the some physical properties of $\mathrm{A}^{\mathrm{II}} \mathrm{B}^{\mathrm{VI}}$ and $\mathrm{A}^{\mathrm{III}} \mathrm{B}^{\mathrm{V}}$ groups of semiconductors.

Reddy et al. [13] have proposed a relationship between a several physical parameters and the refractive index for the $\mathrm{A}^{\mathrm{II}} \mathrm{B}^{\mathrm{VI}}$ and $\mathrm{A}^{\mathrm{III}} \mathrm{B}^{\mathrm{V}}$ groups of semiconductors. Kumar and Singh [14] gave a relationship between an energy gap $E_{\mathrm{g}}$ and the refractive index $n$ for some mixed materials. There are various empirical and semi-empirical rules and expressions that relate $n$ to $E_{\mathrm{g}}$. The refractive index of (B3) BBi can be estimated by using the following formula [14].

$n=K \cdot E_{g} C$

Where: $\mathrm{n}$ is the refractive index, $\mathrm{E}_{\mathrm{g}}$ is the average energy gap (in $\mathrm{eV}$ ), $\mathrm{K}$ and $\mathrm{C}$ are the constants, they are equal respectively: 3.3668 and -0.32234 .

In Moss' rule, the refractive index $n$ and the average energy gap $E_{g}$ are related by $n^{4} E_{g}=K=$ constant $(\approx 100 \mathrm{eV})$. In the Hervé-Vandamme relationship [15]

$\mathrm{n}^{2}=1+\left[\mathrm{A}_{1} /\left(\mathrm{E}_{\mathrm{g}}+\mathrm{A}_{2}\right)\right]^{2}$

Where: $\mathrm{A}_{1}$ and $\mathrm{A}_{2}$ are constants $\left(\mathrm{A}_{1} \approx 13.6 \mathrm{eV}\right.$ and $\left.\mathrm{A}_{2} \approx 3.4 \mathrm{eV}\right)$.

Anani et al [16] reported that, the refractive index of a semiconductor is totally dependent on its bandgap, and they have proposed a linear relation between the refractive-index and the band gap energy $\mathrm{E}_{\mathrm{g}}$, it is given by the following formula:

$n=(17-E g) / 5$

The values of refractive index $\mathrm{n}$ of this compound obtained from the relations of equations (2) and (3) and the value $0.73 \mathrm{eV}$ of the average energy gap obtained initially from first principles calculations are equal to: 3.441 and 3.254 respectively, they are relatively lower than the value of 4.39 obtained by Yalcin et al [17].

The refractive index versus the plasmon energy $\left(\hbar \omega_{\mathrm{p}}\right)$, the force constants $(\alpha$ and $\beta)$ and the lattice energy (U) for some groups $\mathrm{A}^{\mathrm{II}} \mathrm{B}^{\mathrm{VI}}$ and $\mathrm{A}^{\mathrm{III}} \mathrm{B}^{\mathrm{V}}$ semiconductors can be given by the following empirical relations [13]:

$\hbar \omega_{\mathrm{p}}(\mathrm{eV})=\mathrm{K}_{1} \exp \left(\mathrm{K}_{2} \mathrm{n}\right)$

$\alpha(\mathrm{N} / \mathrm{m})=\mathrm{K}_{3} \exp \left(\mathrm{K}_{4} \mathrm{n}\right)$

$\beta(\mathrm{N} / \mathrm{m})=0.28\left(1-\mathrm{f}_{\mathrm{i}}\right) \alpha$

$\mathrm{U}(\mathrm{kcal} / \mathrm{mol})=\mathrm{K}_{5}+\mathrm{K}_{6} \exp \left(\mathrm{K}_{7} \mathrm{n}\right)-\mathrm{K}_{8} \exp \left(\mathrm{K}_{9} \mathrm{n}\right)$

The relevant values of the different constants $\mathrm{K}_{1}, \mathrm{~K}_{2}, \mathrm{~K}_{3}, \mathrm{~K}_{4}, \mathrm{~K}_{5}, \mathrm{~K}_{6}, \mathrm{~K}_{7}, \mathrm{~K}_{8}$ and $\mathrm{K}_{9}$ for $\mathrm{A}^{\mathrm{III}} \mathrm{B}^{\mathrm{V}}$ group semiconductors are respectively: $47.924,-0.3546,286.3,-0.6028,421.224,616.88,-0.1779,86.771$ and $-0.3558[13]$.

The results obtained of the plasmon energy, force constants and lattice energy is given in table 1. Unfortunately, to the best of our knowledge, there are no data available on these parameters for this compound.

S K Gorai and P Mahto [18] have proposed two simple empirical relations to estimate the lattice energy for some binary semiconductors of $\mathrm{A}^{\mathrm{I}} \mathrm{B}^{\mathrm{VII}}, \mathrm{A}^{\mathrm{II}} \mathrm{B}^{\mathrm{VI}}$ and $\mathrm{A}^{\mathrm{III}} \mathrm{B}^{\mathrm{V}}$ groups using the plasmon energy and the bond length. They have observed that the ionic $\mathrm{A}^{\mathrm{III}} \mathrm{B}^{\mathrm{V}}$ compound semiconductors exhibit the highest values of lattice energy, and the $\mathrm{A}^{\mathrm{I}} \mathrm{B}^{\mathrm{VII}}$ alkali halides, being the most ionic, have low values of lattice energy. The $\mathrm{A}^{\mathrm{II}} \mathrm{B}^{\mathrm{VI}}$ compound semiconductors have the intermediate values of both ionicity and lattice energy [18]. 
Table 1: Some Physical Parameters of (B3) BBi Compound. ${ }^{\mathrm{a}}$ Using $\mathrm{n}=3.441,{ }^{\mathrm{b}}$ Using $\mathrm{n}=3.254$

\begin{tabular}{ccc}
\hline Parameter & Value \\
\hline$\hbar \omega_{\mathrm{p}}(\mathrm{eV})$ & $14.146^{\mathrm{a}}$ & $15.115^{\mathrm{b}}$ \\
$\alpha(\mathrm{N} / \mathrm{m})$ & $35.974^{\mathrm{a}}$ & $40.267^{\mathrm{b}}$ \\
$\beta(\mathrm{N} / \mathrm{m})$ & $6.124^{\mathrm{a}}$ & $6.855^{\mathrm{b}}$ \\
$\mathrm{U}(\mathrm{kcal} / \mathrm{mol})$ & $730.18^{\mathrm{a}}$ & $739.74^{\mathrm{b}}$ \\
\hline
\end{tabular}

The plasmon energy, versus the bond length $\mathrm{d}$ for some groups III-V semiconductor materials, can be given by the following relation [19].

$\mathrm{d}(\AA)=\mathrm{C}\left(\hbar \omega_{\mathrm{p}}\right)^{-2 / 3}$

The relevant value of the constant $C$ for $\mathrm{A}^{\mathrm{III}} \mathrm{B}^{\mathrm{V}}$ groups is: 15.30 [19]. Reciprocally to the formula of Eq. (8), we can obtain approximately the following expression:

$\hbar \omega_{\mathrm{p}}=(\mathrm{C} / \mathrm{d})^{3 / 2}$

The results for: the plasmon energy obtained from the relation of equation (9) by using the bond length $d(2.3357 \AA$ ) obtained initially from first principles calculations is equal to: $16.765 \mathrm{eV}$. This value is generally in agreement with the previous results (14.146eV and $15.115 \mathrm{eV}$ ) obtained from the application of Eq. (4).

The ionicity $f_{i}$ of a semiconductor is one of the most fundamental and important physical factors, it can be defined from several models. For example, in the model of Phillips and Van Vechten, the evaluation of the ionicity is based on the dielectric properties of the material. In this model, for tetrahedrally coordinated semiconductors (diamond and zincblende crystal structures), the chemical bonding is considered to be the sum of covalent and ionic contributions [19]. The values of the ionicity obtained by the model of Phillips and Van Vechten are differing somewhat from those proposed by Pauling, which are based on the thermochemistry properties of solids [19].

\subsection{Homopolar and heteropolar energies, dielectric constant and optical susceptibility}

Recently, Gorai Sanjay Kumar [20] has proposed a relationship between the plasmon energy $\left(\hbar \omega_{\mathrm{p}}\right)$ and the homopolar energy $\left(E_{h}\right)$ for some binary tetrahedral $\left(\mathrm{A}^{\mathrm{II}} \mathrm{B}^{\mathrm{VI}}\right.$ and $\left.\mathrm{A}^{\mathrm{III}} \mathrm{B}^{\mathrm{V}}\right)$ semiconductors and ionic $\left(\mathrm{A}^{\mathrm{I}} \mathrm{A}^{\mathrm{VII}}\right)$ compounds. He found that $\log \left(\mathrm{E}_{\mathrm{h}}\right)$ varied linearly with the variation of $\log \left(\hbar \omega_{\mathrm{p}}\right)$, and he has used the values of the plasmon energy and the electronegativity difference between bonding atoms of compounds to predict the homopolar energy gap $\left(\mathrm{E}_{\mathrm{h}}\right)$ and the total energy gap $\left(\mathrm{E}_{\mathrm{g}}\right)$ of these materials.

The plasmon energy can be also given as function of some others parameters: homopolar energy gap $\left(\mathrm{E}_{\mathrm{h}}\right)$, heteropolar energy gap $\left(\mathrm{E}_{\mathrm{c}}\right)$, ionicity $\left(\mathrm{f}_{\mathrm{i}}\right)$ and static dielectric constant $\left(\varepsilon_{0}\right)$ by the following relations [21].

$$
\begin{aligned}
& \mathrm{E}_{\mathrm{h}}=\mathrm{K}_{10}\left(\hbar \omega_{\mathrm{p}}\right)^{\mathrm{K}_{11}} \\
& \mathrm{E}_{\mathrm{c}}=\mathrm{K}_{12} \exp \left[\mathrm{K}_{13}\left(\hbar \omega_{\mathrm{p}}\right)\right] \\
& \mathrm{f}_{\mathrm{i}}=\mathrm{K}_{14}-\mathrm{K}_{15}\left(\hbar \omega_{\mathrm{p}}\right) \\
& \varepsilon_{0}=\mathrm{K}_{16}-\mathrm{K}_{17}\left(\hbar \omega_{\mathrm{p}}\right)
\end{aligned}
$$

The relevant values of the different constants $\mathrm{K}_{10}, \mathrm{~K}_{11}, \mathrm{~K}_{12}, \mathrm{~K}_{13}, \mathrm{~K}_{14}, \mathrm{~K}_{15}, \mathrm{~K}_{16}$ and $\mathrm{K}_{17}$ for $\mathrm{A}^{\mathrm{III}} \mathrm{B}^{\mathrm{V}}$ groups are respectively: $0.0509,1.6192,0.5093,0.1199,0.1809,-0.0126,24.9501$ and 0.9350 .

The results for: homopolar energy gap $\left(\mathrm{E}_{\mathrm{h}}\right)$, heteropolar energy gap $\left(\mathrm{E}_{\mathrm{c}}\right)$, ionicity $\left(\mathrm{f}_{\mathrm{i}}\right)$ and static dielectric constant $\left(\varepsilon_{0}\right)$, obtained from the relations of equations (13), (14), (15), (16) and the value $(16.765 \mathrm{eV})$ of the plasmon energy are respectively: $4.890 \mathrm{eV}, 3.799 \mathrm{eV}, 0.392$ and 9.275 .

Recently, Verma et al. [22] proposed a simple linear relation between the inverse of the high-frequency and static dielectric constants and the product of ionic charges $(\mathrm{ZaZc})$ of the anion and the cation and the average atomic number (Zav) of constituent atoms of $\mathrm{A}^{\mathrm{II}}-\mathrm{B}^{\mathrm{VI}}$ and $\mathrm{A}^{\mathrm{III}}-\mathrm{B}^{\mathrm{V}}$ semiconductor groups, it is given by the following formula:

$\varepsilon=1 /\left[\mathrm{A}-\mathrm{S}(\mathrm{ZaZc} \mathrm{Zav})^{0.25}\right.$ ]

Where: $\varepsilon$ indicates either $\varepsilon_{\infty}$ or $\varepsilon_{0}$.

The values of the constants $\mathrm{A}$ and $\mathrm{S}$ are respectively: $0.19308 \pm 0.01024$ and $0.00126 \pm 1.53552 \times 10^{-4}$ for the highfrequency dielectric constants $\varepsilon_{\infty}$, and $0.12954 \pm 0.00281$ and $6.79659 \times 10^{-4} \pm 4.21458 \times 10^{-5}$ for the static dielectric constants $\varepsilon_{0}$ [22]. Using eq. (14), the values of $\varepsilon_{\infty}$ and $\varepsilon_{0}$ are respectively: 5.376 and 7.954. This later value of the static dielectric constants $\varepsilon_{0}$ is very lower than the value (9.275) obtained by using the formula of the eq. (13).

The values of high-frequency dielectric constant are very lower than the previously calculated data (19.27) obtained by Yalcin et al [17] and the value (14.49) of Bouamama et al [23].

For linear optical materials at lower light-intensity levels, the macroscopic polarization $\mathrm{P}$ induced by light propagating in the medium is proportional to the electric field E, they are related by the following formula: 
$\mathrm{P}=\varepsilon_{0 \mathrm{f}} \chi_{1} \mathrm{E}$

Where: $\chi_{1}$ is the linear optical susceptibility and $\varepsilon_{0 \mathrm{f}}$ is the optical dielectric constant of free space.

In recent years, Yadav et al. [24] proposed the following relation for the calculation of optical susceptibility $\chi$ for II-VI and III-V group binary semiconductors.

$\chi=\mathrm{K}_{18}\left(\hbar \omega_{\mathrm{p}}\right)^{-1.33}$

The relevant value of the constant $\mathrm{K}_{18}$, for $\mathrm{A}^{\mathrm{III}} \mathrm{B}^{\mathrm{V}}$ groups is: 27.77[24].

Using eq. (16) and the value $(16.765 \mathrm{eV})$ of the Plasmon energy, the value of optical susceptibility is equal to 0.653. Unfortunately, there is no data available in the literature on this parameter for BBi compound.

\subsection{Hardness, Debye temperature and melting temperature}

The hardness is one of the most fundamental physical properties of semiconductor compounds; it's understood as the extent to which a given solid resists both elastic and plastic deformations [25]. In experiment, its numerical value is obtained by the application of a charge (pressing an indenter) into the surface of a solid material and measuring the size or dimensions of the impression. The reproducible relationship of a load to the area of indentation is a measure of hardness, such as found in Vickers, or Knoop hardness testers [24]. Gao et al [26] have given a simple emperical formula to calculate the hardness of an overly covalent crystal using the following expression [25].

$$
H(G P a)=350\left[\left(N_{e}\right)^{2 / 3} e^{-1.191 f_{i}}\right] / d^{2.5}
$$

Where $\mathrm{Ne}\left(\mathrm{Ne}=32 / \mathrm{a}_{0}{ }^{3}\right)$ is the electron density expressed in the number of valence electrons per cubic angstrom, $\mathrm{a}_{0}$ is the lattice constant parameter $\left(a_{0}=4 d / \sqrt{3}\right), d$ is the bond length in angstroms, and $\mathrm{f}_{\mathrm{i}}$ is the ionicity of the chemical bond in a crystal scaled by Phillips [27].

The result of the hardness obtained from the relation of equation (17) by using the bond length $d(2.3357 \AA)$ and the ionicity (0.392) is equal to: $9.11 \mathrm{GPa}$. This value is very near than the value (8.9GPa) obtained by Gao et al [26] for the $\mathrm{GaP}$ compound. Unfortunately, to the best of our knowledge, there are no data available in the literature on these parameters for BBi compound.

Recently, Kumar et al. [28] proposed a linear relation between the Debye temperature $\left(\theta_{D}\right)$ and the plasmon energy $\left(\hbar \omega_{\mathrm{p}}\right)$; it is given by the following formula:

$\theta_{D}=-\mathrm{K}_{19}+\mathrm{K}_{20}\left(\hbar \omega_{\mathrm{p}}\right)$

The values of the constants $\mathrm{K}_{19}$ and $\mathrm{K}_{20}$ are respectively: 800.88 and 77.48 for $\mathrm{A}^{\mathrm{III}-} \mathrm{B}^{\mathrm{V}}$ semiconductors [28]. Using eq. (18) and the value $(15.115 \mathrm{eV})$ of the Plasmon, the value of $\theta_{\mathrm{D}}$ have been calculated, listed in table 2 and compared with the available theoretical data $[5,6,29,30]$. It can also be seen, that our calculated value of the Debye temperature is in good agreement with the earlier calculations; its deviation is about 5\% from the value calculated by Deligöz et al [6].

Another linear relation between the Debye temperature and the melting temperature $T_{m}$ was proposed, it is given by the following formula [28]:

$$
\operatorname{Tm}=\left(\theta_{D}+K_{21}\right) / K_{22}
$$

The values of the constants $K_{21}$ and $K_{22}$ are respectively: 153.40 and 0.354 for $\mathrm{A}^{\mathrm{III}}-\mathrm{B}^{\mathrm{V}}$ semiconductors. The obtained value of $T_{m}$ for (B3) $\mathrm{BBi}$ compound at equilibrium lattice parameter have been listed in table 2 and compared with the available theoretical data [6], [29] and [30].

\begin{tabular}{|c|c|c|c|c|}
\hline Parameter & Our work & & Other works & \\
\hline$\theta_{\mathrm{D}}(\mathrm{K})$ & 370.23 & $331.56[5] \mathrm{GGA}$ & $324.11[29]$ & $324.806[30]$ \\
\hline$T_{m}(\mathrm{~K})$ & 1479.18 & $1522 \pm 300[6]$ & 1467 [29] & $1312.866[30]$ \\
\hline
\end{tabular}

Table 2: Debye Temperature and Melting Temperature of (B3) BBi In Comparison With the Available Theoretical Values [5, 6, 29, 30].

Using eq (19), the value of $T_{m}$ for (B3) BBi compound at equilibrium lattice parameter have been calculated, the result obtained is estimated at $1479.18 \mathrm{~K}$. This value is in excellent agreement with the previous calculations data; it deviates from the value $1522 \pm 300 \mathrm{~K}$ of the others [6] and from the value $1467 \mathrm{~K}$ of the others [29] with about $2.81 \%$ and $0.83 \%$ respectively.

\section{Conclusion}

The near-neighbor distance (bond length) and the average energy gap at zero-pressure of zincblende (B3) phase of BBi compound are initially calculated, then they are used to predict some others optoelectronic and thermal properties . The refractive index, the plasmon energy, the force constants, the lattice energy, the homopolar and heteropolar energies, the ionicity, the linear optical susceptibility, the hardness and the dielectric constant are predicted. 
Unfortunately, to the best of our knowledge, there is no data available in the literature on the majority of these parameters for the BBi compound. Future experimental work or other theoretical calculations will contribute for the validating of our calculated result of these parameters. The Debye temperature and the melting temperature of this material are obtained; they are in general in good agreement with the other theoretical results of the literature.

\section{References}

[1] S. Shinde, M. Talati, K. Jha. Prafulla, \& S. P. Sanyal, "Theoretical study of the transverse acoustic phonons of GaSb at high pressure", Pramana Journal of Physic, Vol.63, No.2, (2004), pp.425-429. http://www.ias.ac.in/pramana/ns/ns60.

[2] A. Zaoui, D. Madouri \& M. Ferhat, "First-principles study of the ground state stability of III-V bismuth compounds", Philosophical Magazine Letters, Vol.89, No.12, (2009), pp.807-813, DOI:10.1080/09500830903304125.

[3] D. Madouri \& M. Ferhat, "How do electronic properties of conventional III-V semiconductors hold for the III-V boron bismuth BBi compound?", Physica Status Solidi (b), Vol.242, No.14, (2005), pp. 2856-2863, DOI: 10.1002/pssb.200441121.

[4] P. Blaha, K. Schwarz, P. Sorantin \& S. B Trickey, "Full-potential, linearized augmented plane wave programs for crystalline systems", Computer Physics Communications. Vol.59, No.2, (1990), pp. 399-415. http://www.readcube.com/articles/10.1016/0010- 4655(90)90187-6.

[5] S. Cui, W. Feng, H. Hu, Z. Feng \& Y. Wang, "First principles studies of phase stability, electronic and elastic properties in BBi compound", Computational Materials Science, Vol.47, No.4, (2010), pp. 968-972. http://www.sciencedirect.com/science/article/pii/S0927025609004418.

[6] E. Deligöz, K. Colakoglu, Y. O. Ciftci, \& H. Ozisik, "The first principles study on boron bismuth compound", Computational Materials Science, Vol.39, No.3, (2007), pp. 533-540. http://www.sciencedirect.com/science/article/pii/S0927025606002321.

[7] S. Singh, \& M. Sarwan, "Structural phase transition and high pressure elastic behavior of BX $(\mathrm{X}=\mathrm{Sb}, \mathrm{Bi})$ compounds", Journal of optoelectronics and advanced materials, Vol.12, No.10, (2010), pp. 2106-2112. http://www. joam.inoe.ro/download.php?idu=2623.

[8] E. Engel \& R. M. Dreizler, "Density Functional Theory", Springer-Verlag, New York, (2011).

[9] X. Gonze, G. M. Rignanese, M. Verstraete, et al. "A brief introduction to the ABINIT software package", Zeitschrift für Kristallographie, Vol.220, No.5-6, (2005), pp. 558-562. dx.doi.org/10.1524/zkri.220.5.558.65066.

[10] S. Goedecker, M. Teter \& J. Hutter, "Separable dual-space gaussian pseudopotentials", Physical Review B, Vol.54, No.3, (1996), pp. 17031710. DOI: http://dx.doi.org/10.1103/PhysRevB.54.1703.

[11] C. Hartwigsen, S. Goedecker \& J. Hutter, "Relativistic separable dual-space gaussian pseudopotentials from H to Rn", Physical Review B, Vol.58, No.7, (1998), pp. 3641-3662, DOI: http://dx.doi.org/10.1103/PhysRevB.58.3641.

[12] H .J. Monkhorst \& J. D. Pack, "Special points for Brillouin-zone integrations", Physical Review B, Vol.13, No.12, (1976), pp. 5188-5192. DOI: http://dx.doi.org/10.1103/PhysRevB.13.5188.

[13] R. R. Reddy, Y. Nazeer Ahammed, P. Abdul Azeem, K. Rama Gopal, B. Sasikala Devi \&T. V. R. Rao, "Dependence of Physical Parameters of Compound Semiconductors on Refractive Index", Defense Science Journal, Vol. 53, No. 3, (2003), pp. 239-248. http://www. publications.drdo.gov.in/ojs/index.php/dsj/article/view/2272.

[14] V. Kumar \& J. K. Singh, "Model for calculating the refractive index of different materials", Indian Journal of Pure and Applied Physics, Vol.48, (2010), pp. 571-574. http://www.nusod.org/piprek/guden96msmse.pdf.

[15] P. J. L. Herve, \& L. K. J. Vandamme, "Empirical temperature dependence of the refractive index of semiconductors", Journal of Applied Physics, Vol. 77, No. 10, (1995) pp. 5476 - 5477. http://www. link.springer.com/content/pdf/10.1007\%2FBF00665719.pdf.

[16] M. Anani, C. Mathieu, S. Lebid, Y. Amar, Z. Chama \& H. Abid, "Model for calculating the refractive index of a III-V semiconductor", Computational Materials Science, Vol.41, No.4, (2008), pp. 570-575. www.sciencedirect.com/science/article/pii/S0927025607001577.

[17] B. G. Yalcin, M. Ustundag \& M. Aslan, "Optical Properties of BN and BBi Compounds", Acta Physica Polonica A, Vol.125, No.2, (2014), pp. 574-576. http://www.przyrbwn.icm.edu.pl/APP/PDF/125/a125z2p131.pdf.

[18] S. K. Gorai \& P. Mahto, "Plasmon energy and lattice energy of binary tetrahedral semiconductors and I-VII ionic compounds", Indian Journal of Physics, Vol.86, No.4, (2012), pp. 273-277. http://www.link.springer.com/content/pdf/10.1007\%2Fs12648-012-0053-y.pdf

[19] J. I. Gersten \& F. W. Smith, "The physics and chemistry of materials", New York: John Wiley \& Sons, (2001).

[20] G. S. Kumar, "Analysis of Ionicity using Plasmon Energy and Electro Negativity Difference of Binary Tetrahedral Semiconductors and Ionic Compounds", Research Journal of Physical Sciences, Vol.1, No.3, (2013), pp. 6-11. www.isca.in/PHY_SCI/Archive/v1/.../2.ISCA-RJPS2013-013.pdf.

[21] V. Kumar, A. K. Shrivastava, Anita Sinha, \& Vijeta Jha, "Dielectric properties of different materials", Indian Journal of Pure and Applied Physics, Vol. 51, No. 1(2013), pp. 49-54. http://www.nopr.niscair.res.in/handle/123456789/15365.

[22] S. Verma, Naresh Pal, B. K. Sarkar, R. Bhandari \& V. K. Jindal, "Dielectric constants of zinc-blende semiconductors", Physica Scripta. Vol. 85, No. 1 (2012), 015705 (4pp). DOI:10.1088/0031-8949/85/01/015705.

[23] Kh. Bouamama, P. Djemia, N. Lebgaa \& K. Kassali, "Ab initio calculation of the lattice dynamics of the Boron group-V compounds under high pressure", High Pressure Research, Vol. 27, No. 2 (2007), pp. 269 -277. DOI: 10.1080/08957950701265359

[24] D. S. Yadav, C. kumar, J. Sigh, Parashuram \& G. Kumar, "Optoelectronic properties of zinc blende and wurtzite structured binary solids", Journal of Engineering and Computer Innovations, Vol. 3, No. 2, (2012), pp. 26-35. http://www.academicjournals.org/journal/JECI/articleabstract/DC4DD528645.

[25] A. Šimůnek \& J. Vackář, " Hardness of Covalent and Ionic Crystals: First-Principle Calculations", Physical Review Letters, Vol. 96, (2006), 085501 (4pp). DOI: http://dx.doi.org/10.1103/PhysRevLett.96.085501

[26] F. M. Gao, J. L. He, E. D. Wu, S. M. Liu, D. L. Yu, D. C. Li, S. Y. Zhang, \& Y. J. Tian, "Hardness of Covalent Crystals", Physical Review Letters, Vol. 91, No.1 (2003), 015502 (4pp). DOI: http://dx.doi.org/10.1103/PhysRevLett.91.015502.

[27] J. C. Phillips, "Ionicity of the Chemical Bond in Crystals", Reviews of Modern Physics, Vol. 42, No.3 (1970), pp. 317-356, DOI: http: //dx.doi.org/10.1103/RevModPhys.42.317.

[28] V. Kumar, V. Jha, \& A. K. Shrivastava, "Debye temperature and melting point of II-VI and III-V semiconductors", Crystal Research and Technology, Vol. 45, No. 9, (2010) pp. 920-924. http://www. onlinelibrary.wiley.com/doi/10.1002/crat.201000268/pdf.

[29] K. Amara, B. Soudini, D. Rached \& A. Boudali, "Molecular dynamics study on thermomechanical properties of cubic BBi", Computational Materials Science, Vol. 44, No. 2, (2008) pp. 635-640. DOI: 10.1016/j.commatsci.2008.04.023.

[30] M. Ustundag, M. Aslan, \& Battal G. Yalcin, "The first-principles study on physical properties and phase stability of Boron-V (BN, BP, BAs, BSb and BBi) compounds", Computational Materials Science, Vol. 81, (2014) pp. 471-477. http://dx.doi.org/10.1016/j.commatsci.2013. 08.056 . 